\title{
El GES promueve la equidad en el tratamiento del gran quemado de la tercera edad
}

\author{
CLAUDIA R. ALBORNOZ G., JORGE VILLEGAS C., \\ ISIDE BRAVO Y., VERÓNICA PEÑA M.
}

\section{Analysis of the explicit guarantees of health inclusion criteria for elderly burned patients}

Background: The guidelines for the treatment of severely burned patients, included in the explicit guarantees in health care (GES), accept having a Garces' index over 70, among others, as an inclusion criterion. This criterion allows elderly patients with small total burn surface area (TBSA) to have access to GES. Aim: To analyze if a universal access to GES for this group of patients is justified. Material and Methods: Revision of medical records of adult patients admitted to a burn service. Causative agent, TBSA, associated illnesses and outcome were compared between 218 subjects aged 65 years or more and 720 subjects aged less than 65 years. Results: Older subjects had smaller TBSA, a lower prevalence of inhalation injury and more associated diseases. Their lethality was three times greater than that of younger subjects and their risk of dying. When adjusting for TBSA, presence of inhalation injury and associated diseases was 11 times greater. Conclusions: The inclusion of older people with lower TBSA in the explicit guarantees in health is fully justified, considering the lethality of burns in this age group.

(Rev Med Chile 2011; 139: 1465-1470).

Key words: Aged; Burns; Delivery of health care.

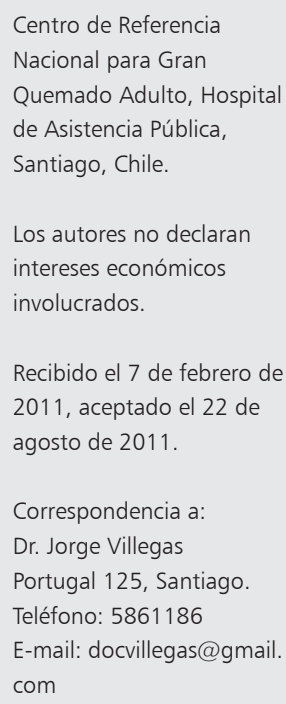

E 1 año 2007, el plan de Garantías Explícitas en Salud (GES) incluyó al tratamiento del paciente gran quemado. Los criterios de inclusión en el GES ${ }^{1}$ se basan, entre otros, en el índice de gravedad de Garcés ${ }^{2}$ que se calcula con la fórmula presentada en la Figura 1.

De acuerdo al puntaje obtenido categoriza a los pacientes en grupos de gravedad y pronostica porcentajes de letalidad ${ }^{2}$. Al analizar la Guía Clínica del gran quemado ${ }^{1}$, se evidencia que el paciente gran quemado añoso está cubierto casi universalmente, independiente de la extensión de la quemadura. Con un índice de gravedad (IG) mayor a 70 el paciente recibe los beneficios del GES, que incluyen el traslado al Centro de Referencia Nacional dentro de las primeras 72 horas, tratamiento protocolizado y protección financiera. Para los adultos mayores es fácil obtener este puntaje, ya que un paciente de 65 años con una quemadura pequeña, de $3 \%$, tendrá un IG de 71.

El envejecimiento de Chile, y los cambios en su estructura poblacional de una pirámide constrictiva a una estacionaria, llevarán a que el año 2020 haya más de dos millones de adultos mayores, según proyecciones del Instituto Nacional de Estadísticas ${ }^{3}$. Este aumento de la población mayor de 65 años puede llevar a un aumento del número de quemados añosos, que ya en los 3 primeros años de funcionamiento del Centro de Referencia Nacional correspondió al 30\% del total de los pacientes que requirieron de cuidados intermedios o intensivos (Datos no publicados). En la literatura internacional, los pacientes quemados añosos corresponden al 7-20\% del total de ingresos a los centros de quemados.

Los adultos mayores tienen menos reservas 
Índice de Gravedad de Garcés (IG)= Edad (años) + \% SCQ A x $1+\%$ SCQ AB $\times 2+\%$ SCQ B $\times 3$

$\%$ SCQ A: \% de superficie quemada superficial

$\%$ SCQ AB: \% de superficie quemada intermedia o de espesor parcial.

$\%$ SCQ B: \% de superficie quemada profunda o de espesor total.
Figura 1. Fórmula para calcular el Índice de Garcés. funcionales, tanto por la mayor cantidad de patologías crónicas como el envejecimiento en sí mismo ${ }^{5-7}$. Un paciente quemado añoso tiene menos capacidad de respuesta ante la injuria que un paciente más joven, es por eso que ante un accidente como una quemadura, tiene menos posibilidades de sobrevida ${ }^{5,8-10}$.

El objetivo de este trabajo es evaluar si se justifica esta discriminación positiva o universalidad para la inclusión del paciente gran quemado añoso en el GES.

\section{Material y Métodos}

Se diseñó un estudio de cohorte histórica de los pacientes ingresados al Centro de Referencia Nacional para gran quemado adulto (Servicio de Quemados, Hospital de Asistencia Pública Alejandro del Río) entre los años 2006 y 2010 . Se definió adulto como pacientes mayores de 15 años. Los criterios de inclusión en la cohorte correspondieron a la definición de "gran quemado" establecidos por las guías clínicas del GES:

- Pacientes con Índice de gravedad de Garcés $>70$ puntos o con quemaduras $\mathrm{AB}$ o $\mathrm{B}>20 \%$ de SCQ.

- Pacientes $>65$ años con $10 \%$ o más de quemadura $\mathrm{AB}$ o $\mathrm{B}$.

- Pacientes con quemaduras respiratorias/por inhalación de humo.

- Pacientes con quemaduras eléctricas de alta tensión.

- Pacientes quemados politraumatizados.

- Pacientes quemados con patologías graves asociadas.

- Pacientes con quemaduras térmicas.

La cohorte incluyó al universo de pacientes ingresados durante el período descrito, analizándose un total de 938 pacientes. Se consideró "expuestos" a los 218 pacientes mayores de 65 años, decidién- dose éste punto de corte por corresponder a la clasificación de tercera edad de la Organización Mundial de la Salud ${ }^{11}$. Se comparó con la cohorte de pacientes quemados menores de 65 años $(\mathrm{n}=$ 720) hospitalizados durante el mismo período en el mismo centro. Los datos fueron obtenidos del registro electrónico creado por el Dr. Omar Atenas y complementados con revisión de los registros clínicos y la base de datos de egresos de la Asistencia Pública.

Se consideró como desenlace el fallecimiento intrahospitalario durante el ingreso causado por la quemadura. Se analizaron las variables: agente productor de la quemadura, presencia de injuria inhalatoria, presencia de comorbilidades (hipertensión, diabetes, cardiopatía, enfermedad pulmonar obstructiva crónica, daño hepático crónico, alcoholismo, epilepsia, desnutrición, obesidad, alteraciones psiquiátricas, neurológicas, abandono social), superficie corporal quemada total y profunda y dosis letal 50 (DL50) que es un indicador usado en pacientes quemados que evalúa la superficie corporal quemada en que se producen el $50 \%$ de las muertes.

Las variables continuas (edad, superficie corporal quemada) se expresaron en promedio y desviación estándar y las categóricas (agente, presencia de comorbilidades, injuria inhalatoria) en porcentajes y proporciones. Las variables superficie corporal quemada y número de cirugías no distribuyen normalmente por lo que se usó para su comparación test no paramétrico (Mann Whitney). Para comparación de proporciones (género y agente) se usó proportion test. Para la comparación de variables dicotómicas en ambos grupos (agente, comorbilidades, injuria inhalatoria) se usó Test de Fisher o $\chi^{2}$ según necesidad. La asociación entre la exposición a "mayor edad" y letalidad se evaluó con análisis multivariado, incluyendo en el modelo variables que han demostrado asociación a letalidad en la literatura (injuria inhalatoria, superficie corporal quemada 
y comorbilidades $)^{12-28}$. Se consideraron significativos valores de $\alpha<0,05$ y Odds Ratio mayor a 1,00 con intervalos de confianza que no incluyeran al valor nulo. El análisis estadístico se realizó con el programa computacional Stata ${ }^{\circledR}$, versión 11.

\section{Resultados}

Entre los años 2006 y 2010, ingresaron 938 pacientes al Centro de Referencia nacional para el quemado grave, de los cuales 218 (23\%) correspondieron a pacientes de la tercera edad. La Tabla 1 muestra las características principales de cada grupo. La edad promedio del grupo no expuesto fue de 38, 2 años y la del grupo expuesto 76,7 años. Los pacientes mayores tienen predominio de género femenino $(\mathrm{p}<0,001)$, tienen más frecuentemente comorbilidades $(\mathrm{p}<0,001)$ y menor prevalencia de injuria inhalatoria $(\mathrm{p}<0,001)$.

La superficie corporal quemada fue significativamente menor en los ancianos con un promedio de $13,5 \%$ versus los más jóvenes con $26 \%$ ( $\mathrm{p}<$ $0,001)$. La superficie quemada profunda en el momento del ingreso, es similar en ambos grupos (7\%). La proporción del total de la quemadura que es profunda desde un principio es de 0,58 en los pacientes añosos, comparado con 0,27 en los más jóvenes.

La Tabla 2 muestra la frecuencia de los distintos agentes productores de la quemadura. En la mayoría de los pacientes de ambos grupos el agente predominante es el fuego, siendo en ancianos responsable de $59 \%$ de los casos. Las quemaduras por líquidos calientes (escaldaduras) son significativamente más frecuentes en el grupo de adultos mayores siendo la segunda causa con $28 \%$ de los casos ( $\mathrm{p}<0,001)$. No se encontró diferencias significativas en la proporción de otros agentes. Hubo 2 quemaduras por contacto con planchas. En los más jóvenes otras causas toman importancia como las quemaduras eléctricas. $86 \%$ de las quemaduras del grupo de pacientes de tercera edad se produjeron en domicilio y sólo 3,2\% fueron relacionadas con algún tipo de trabajo. Hubo 2 pacientes mayores en que la quemadura fue auto inferida, y 4 pacientes en que se debió a una agresión (diferentes a los pacientes con quemaduras de contacto con una plancha).

En los pacientes del grupo expuesto las comorbilidades son significativamente más frecuentes, $73,4 \%$ comparado con $38,5 \%$ del grupo de pacientes más jóvenes. Los pacientes añosos tienen más frecuentemente hipertensión (48\%), diabetes $(18,3 \%)$, cardiopatía $(14,2 \%)$ y enfermedad pulmonar crónica $(6 \%)$, todas estadísticamente significativas. En los pacientes más jóvenes fue significativamente más frecuente el alcoholismo y epilepsia (Tabla 3). Veinticuatro adultos mayores (11\%) tenían algún factor que determinaba serias dificultades para la rehabilitación posterior (caso social, ceguera, daño orgánico cerebral, enfermedad psiquiátrica, secuela neurológica).

Al analizar la mortalidad, ésta es significativamente mayor en el grupo expuesto, $36,4 \%$ versus $13,4 \%(\mathrm{p}<0,001)$. La probabilidad de morir de un paciente añoso es 10,8 veces mayor que la de uno más joven, ajustado por superficie corporal, injuria inhalatoria y comorbilidades (Tabla 4). Eso significa que a una misma superficie corporal quemada, injuria inhalatoria y presencia de comorbilidades, los mayores tienen casi 11 veces más

Tabla 1. Comparación de características principales de pacientes menores y mayores de 65 años, ingresados en el Centro de Referencia Nacional para Quemado adulto durante 2006 y 2010

\begin{tabular}{|lccr|}
\hline & $\begin{array}{c}\text { Grupo “no expuesto” } \\
(<\mathbf{6 5} \text { años) }\end{array}$ & $\begin{array}{c}\text { Grupo “expuesto” } \\
\text { ( } \mathbf{6} \mathbf{6 5} \text { años) }\end{array}$ & p \\
\hline Edad (promedio, DE) & $38,2(13,0)$ & $76,7(7,5)$ & $<0,001$ \\
Género femenino (\%) & 29,0 & 53,0 & $<0,001$ \\
\hline Presencia de Comorbilidades (\%) & 38,5 & 73,4 & $<0,001$ \\
\hline Superficie corporal quemada (promedio, DE) & $25,8( \pm 21)$ & $13,5( \pm 14,8)$ & 0,07 \\
\% Superficie corporal quemada profunda (promedio, DE) & $7,1( \pm 13,5)$ & $7,9( \pm 13,3)$ & 0,001 \\
\hline Injuria inhalatoria (\%) & 24,0 & 16,0 & \\
\hline
\end{tabular}

DE: desviación estándar. 
Tabla 2. Frecuencia de agentes productores de quemadura en pacientes menores y mayores de 65 años

\begin{tabular}{|lcccc|}
\hline Agente & & $\begin{array}{c}\text { Grupo } \\
\text { "no expuesto" } \\
\text { (<65 años) }\end{array}$ & $\begin{array}{c}\text { Grupo } \\
\text { "expuesto" } \\
\text { ( } \geq \mathbf{6 5} \text { años) }\end{array}$ & p \\
\hline Fuego & $\%(n)$ & $59(428)$ & $55(120)$ & 0,87 \\
\hline Escaldadura & $\%(n)$ & $13,4(97)$ & $28(61)$ & $<0,001$ \\
\hline Explosión & $\%(n)$ & $8,3(60)$ & $8,2(18)$ & 0,5 \\
Electricidad & $\%(n)$ & $7,3(53)$ & $0,92(2)$ & 0,99 \\
Químico & $\%(n)$ & $1,6(12)$ & 0 & 0,9 \\
Contacto & $\%(n)$ & $0,8(6)$ & $0,9(2)$ & 0,5 \\
\hline Desconocido & $\%(n)$ & $0,56(4)$ & $6,8(15)$ & 0,8 \\
\hline
\end{tabular}

La primera causa de quemaduras en pacientes añosos es el fuego, seguido por líquidos calientes.

Tabla 3. Frecuencia de comorbilidades en pacientes mayores y menores de 65 años

\begin{tabular}{|c|c|c|c|c|}
\hline Comorbilidad & & $\begin{array}{c}\text { Grupo } \\
\text { "no expuesto" } \\
\text { (< } 65 \text { años) }\end{array}$ & $\begin{array}{c}\text { Grupo } \\
\text { "expuesto" } \\
\text { ( } \geq 65 \text { años) }\end{array}$ & $\mathbf{p}$ \\
\hline Hipertensión & (\%) & 7,3 & 48,1 & $<0,001$ \\
\hline Diabetes mellitus & (\%) & 4,7 & 18,3 & $<0,001$ \\
\hline Cardiopatía & (\%) & 1,25 & 14,2 & $<0,001$ \\
\hline $\begin{array}{l}\text { Enfermedad } \\
\text { pulmonar } \\
\text { crónica }\end{array}$ & (\%) & 1,1 & 6 & $<0,001$ \\
\hline Alcoholismo & (\%) & 12,6 & 6,4 & 0,005 \\
\hline Desnutrición & (\%) & 0 & 2,7 & $<0,001$ \\
\hline Obesidad & (\%) & 7,7 & 10 & 0,2 \\
\hline Epilepsia & (\%) & 6,11 & 2,7 & 0,002 \\
\hline $\begin{array}{l}\text { Daño hepático } \\
\text { crónico }\end{array}$ & $(\%)$ & 0,14 & 0,9 & 0,06 \\
\hline
\end{tabular}

Tabla 4. Análisis multivariado del riesgo de morir en pacientes mayores de 65 años comparado con menores de 65 años, ajustado por superficie corporal quemada, injuria inhalatoria y presencia de comorbilidades

\begin{tabular}{|lcc|}
\hline Variable independiente & Odds Ratio & $\begin{array}{c}\text { Intervalo de } \\
\text { confianza al 95\% }\end{array}$ \\
\hline Edad mayor de 65 años & 10,84 & $5,57-21,0$ \\
\hline Superficie corporal quemada & 1,07 & $1,05-1,08$ \\
\hline Injuria inhalatoria & 2,09 & $1,20-3,66$ \\
\hline Presencia de comorbilidades & 1,91 & $1,08-3,36$ \\
\hline
\end{tabular}

riesgo de morir. La Dosis letal 50, que es la superficie corporal quemada en la que se produce $50 \%$ de las muertes, es de $14 \%$ de superficie corporal quemada en los ancianos, inferior al 49,8\% de los más jóvenes.

No hubo sobrevivientes sobre los 85 años de edad, independiente de la superficie corporal quemada.

\section{Discusión}

Existe efectivamente un sesgo de selección que favorece el ingreso de los pacientes añosos con quemaduras menos extensas al GES. Sin embargo, en vista de la evidencia aquí expuesta: riesgo casi 11 veces más alto de morir y mayor número de comorbilidades, creemos que esta discriminación positiva se justifica plenamente, apoyado por el mayor riesgo que presentan estos pacientes. A una misma superficie corporal quemada, injuria inhalatoria y comorbilidades, los pacientes añosos tienen 11 veces más riesgo de morir que los más jóvenes. La mitad de las muertes se produce en 14\% de superficie quemada en adultos mayores y casi $50 \%$ en los más jóvenes, lo que refleja la vulnerabilidad de estos pacientes. Todos estos hechos representan la evidencia necesaria para apoyar la incorporación universal de los pacientes de la tercera edad al GES, aunque sus superficies corporales quemadas sean menos extensas y tengan menos prevalencia de injuria inhalatoria.

Este elevado riesgo de morir puede explicarse en parte por reservas funcionales disminuidas, por un aumentado número de comorbilidades ${ }^{5,10,29-31}$, por una menor posibilidad de reaccionar ante el peligro, menor capacidad de reacción ante el estrés, por tener piel más atrófica ${ }^{5-9}$ lo que hace que la proporción de la quemadura profunda sea mayor desde el ingreso y a que desarrollan más frecuentemente complicaciones intrahospitalarias como edema pul- 
monar, neumonía e insuficiencia cardiaca congestiva $^{5,8,9}$. Otra explicación podría ser la presencia de una mayor proporción de mujeres quemadas en este grupo etáreo; el género femenino ha sido demostrado previamente como un factor de riesgo de morir en pacientes quemados, pese a que no se ha elucidado el mecanismo ${ }^{13-16,25,32}$. El GES ha contribuido a la equidad favoreciendo el ingreso de pacientes que si se considerara sólo la superficie corporal quemada no podrían hacerlo, privilegiando a la mayor edad y reconociendo que es una eventual fuente de mayores complicaciones.

Además de la oportunidad de tratamiento adecuado, es muy importante también poner énfasis en la prevención. La gran mayoría de las quemaduras tanto en nuestro estudio como en la literatura se produce en el domicilio de los pacientes $^{5-8,31,33-35}$. La mayoría de las veces estos accidentes son prevenibles, y al igual que con los niños, debería evitarse la manipulación de fuego en pacientes añosos y cautelar el uso de líquidos calientes, por ejemplo, recipientes con agua hirviendo para el aseo personal o para cocinar. Las familias deberían estar informadas y ser concientes de este riesgo para poder tomar las precauciones necesarias.

Los pacientes añosos pueden tener una rehabilitación y reinserción más dificultosa al tener menores reservas funcionales, nutricionales y menor red de apoyo ${ }^{7,33,34}$. Este punto también debe ser tomado en cuenta para apoyar estrategias de prevención en este grupo etáreo y para favorecer la entrega de tratamiento oportuno con el objetivo de lograr la reparación de la barrera cutánea lo antes posible, y así evitar complicaciones inmediatas como la muerte o a más largo plazo como secuelas y discapacidad. En este estudio se encontró 11\% de pacientes que presentaban al menos una condición que potencialmente podría dificultar la rehabilitación, sin considerar la falta de redes sociales.

Como conclusión, los pacientes mayores de 65 años, a pesar de tener menores superficies quemadas y menor injuria inhalatoria, tienen una letalidad que casi triplica la de los más jóvenes y un riesgo de morir 11 veces mayor. Dados los cambios demográficos que está viviendo nuestro país, es muy importante entregar especial atención y prevención a este grupo que es más vulnerable. El GES, al incluir a casi el 100\% de los adultos mayores con quemaduras, ha contribuido a mejorar la equidad para este grupo de pacientes que si bien tienen quemaduras más pequeñas no cuenta con los mecanismos adecuados para defenderse apropiadamente.

Agradecimientos: A los doctores Omar Atenas y Reinaldo Martínez por su ayuda en la recolección de los datos.

\section{Referencias}

1. Chile. Ministerio de Salud. Guía Clínica Gran Quemado. 2007.

2. Garcés M, James E, Castillo E, Morales J, Salas CEC: Bases Clínicas y Epidemiológicas para un pronóstico del Enfermo Quemado. Revista de la Asistencia Pública 1971 (6): 9-14.

3. INE. Chile hacia el 2050: Proyecciones de población. Publicación del Instituto Nacional de Estadísticas 2005, 1: 1-30.

4. Macrino S, Slater H, Aballay A, Goldfarb IW, Caushaj PF. A three-decade review of thermal injuries among the elderly at a regional burn centre. Burns 2008; 34 (4): 509-11.

5. Mabrouk A, Maher A, Nasser S: An epidemiologic study of elderly burn patients in Ain Shams University Burn Unit, Cairo, Egypt. Burns 2003; 29 (7): 687-90.

6. Keck M, Lumenta DB, Andel H, Kamolz LP, Frey M: Burn treatment in the elderly. Burns 2009; 35 (8): 10719.

7. Huang SCW, Huang C, Tsai C. Management of elderly burn patients. Int J Gerontol 2008; 2 (3): 91-7.

8. Ho WS, Ying SY, Chan HH. A study of burn injuries in the elderly in a regional burn centre. Burns 2001; 27 (4): 382-5.

9. Lionelli GT, Pickus EJ, Beckum OK, Decoursey RL, Korentager RA. A three decade analysis of factors affecting burn mortality in the elderly. Burns 2005; 31(8): 958-63.

10. Rao K, Ali SN, Moiemen NS. Aetiology and outcome of burns in the elderly. Burns 2006; 32 (7): 802-5.

11. Organización Panamericana para la Salud. Mejoremos la salud a todas las edades: Un manual para el cambio de comportamiento. 2005:147-160 [Disponible en http:// publicationspahoorg/spanish/PC_590_Tercera_edadpdf, consultado 1 de febrero de 2011].

12. Zoch G, Schemper M, Kyral E, Meissl G. Comparison of prognostic indices for burns and assessment of their accuracy. Burns 1992; 18 (2): 109-12.

13. Tobiasen J, Hiebert JM, Edlich RF. The abbreviated burn severity index. Ann Emerg Med 1982; 11 (5): 260-2.

14. Germann G, Barthold U, Lefering R, Raff T, Hartmann $\mathrm{B}$. The impact of risk factors and pre-existing conditions 
on the mortality of burn patients and the precision of predictive admission-scoring systems. Burns 1997; 23 (3): 195-203.

15. O'Keefe GE, Hunt JL, Purdue GF. An evaluation of risk factors for mortality after burn trauma and the identification of gender-dependent differences in outcomes. J Am Coll Surg 2001; 192 (2): 153-60.

16. Muller MJ, Pegg SP, Rule MR. Determinants of death following burn injury. Br J Surg 2001; 88 (4): 583-7.

17. Ho WS, Ying SY, Burd A: Outcome analysis of 286 severely burned patients: retrospective study. Hong Kong Med J 2002; 8 (4): 235-9.

18. Brusselaers N, Monstrey SJ, Vandijck DM, Blot SI. Prediction of morbidity and mortality on admission to a burn unit. Plast Reconstr Surg 2007; 120 (1): 360-1; author reply 361 .

19. McGwin G Jr, George RL, Cross JM, Rue LW. Improving the ability to predict mortality among burn patients. Burns 2008; 34 (3): 320-7.

20. Burn injury group: Development and validation of a model for prediction of mortality in patients with acute burn injury. Br J Surg 2009; 96 (1): 111-7.

21. Ryan CM, Schoenfeld DA, Thorpe WP, Sheridan RL, Cassem EH, Tompkins RG. Objective estimates of the probability of death from burn injuries. N Engl J Med 1998; 338 (6): 362-6.

22. Zawacki BE, Azen SP, Imbus SH, Chang YT. Multifactorial probit analysis of mortality in burned patients. Ann Surg 1979; 189 (1): 1-5.

23. Coste J, Wasserman D, Venot A: Predicting mortality in adult burned patients: methodological aspects of the construction and validation of a composite ratio scale. J Clin Epidemiol 1996; 49 (10): 1125-31.

24. Gómez-Cia T, Mallen J, Marquez T, Portela C, López I. Mortality according to age and burned body surface in the Virgen del Rocio University Hospital. Burns 1999; 25 (4): 317-23.

25. Galeiras R, Lorente JA, Pertega S, Vallejo A, Tomicic V, de la Cal MA, et al. A model for predicting mortality among critically ill burn victims. Burns 2009; 35 (2): 201-9.

26. Shirani KZ, Pruitt BA, Jr, Mason AD Jr. The influence of inhalation injury and pneumonia on burn mortality. Ann Surg 1987; 205 (1): 82-7.

27. Tredget EE, Shankowsky HA, Taerum TV, Moysa GL, Alton JD. The role of inhalation injury in burn trauma. A Canadian experience. Ann Surg 1990; 212 (6): 720-7.

28. Thombs BD, Singh VA, Halonen J, Diallo A, Milner SM. The effects of preexisting medical comorbidities on mortality and length of hospital stay in acute burn injury: evidence from a national sample of 31,338 adult patients. Ann Surg 2007; 245 (4): 629-34.

29. Li X, Peng Y, Shang X, Liu S. Epidemiologic investigation of geriatric burns in Southwest China. Burns 2009; 35 (5): 714-8.

30. Khadim MF, Rashid A, Fogarty B, Khan K. Mortality estimates in the elderly burn patients: the Northern Ireland experience. Burns 2009; 35 (1): 107-13.

31. Wibbenmeyer LA, Amelon MJ, Morgan LJ, Robinson BK, Chang PX, Lewis R, 2nd, Kealey GP. Predicting survival in an elderly burn patient population. Burns 2001; 27 (6): 583-90.

32. Kerby JD, McGwin G Jr, George RL, Cross JA, Chaudry $\mathrm{IH}$, et al. Sex differences in mortality after burn injury: results of analysis of the National Burn Repository of the American Burn Association. J Burn Care Res 2006; 27 (4): 452-6.

33. Wong P, Choy VY, Ng JS, Yau TT, Yip KW, Burd A. Elderly burn prevention: a novel epidemiological approach. Burns 2007; 33 (8): 995-1000.

34. Lumenta DB, Hautier A, Desouches C, Gouvernet J, Giorgi R, Manelli JC, et al. Mortality and morbidity among elderly people with burns-evaluation of data on admission. Burns 2008; 34 (7): 965-74.

35. Yin Z, Qin Z, Xin W, Gómez M, Zhenjiang L. The characteristics of elderly burns in Shanghai. Burns; 36 (3): 430-5. 